held by its present defenders (and upon any three-color theory), it is blue-green; the whole bearing of the case upon existing theories depends upon whether this color (the complement to the fundamental red of both theories) is properly named green or blue-green; in other words, it has no bearing at all upon them. König has very recently had several most interesting cases of monocular blue-blindness circumscribed within a small area surrounding the fovea, in which the sensation given by the cold end of the spectrum can be readily compared with normal sensations, and is found to be bluegreen. This fact is still, of course, not decisive between theories, for what Hering means by green is a color which is pronounced to be blue-green by the ordinary consciousness, and this in spite of the fact that it is precisely for the satisfaction of the ordinary consciousness as regards color-sensation that Hering's theory has been devised.

C. Ladi Franklin.

\title{
SENSATIONS OF TASTE.
}

Contributions to the Physiological Psychology of the Sense of Tasic. FRIEDRICH Kiesow. Abstract of papers in Philosophische Studien, Bd. X, Heft 3, pp. 329 ff; Heft 4, pp. $5^{23}$ ff.

The electric, metallic and alkaline tastes being reserved for special investigations, the present work treats only of those sensations recognised as special qualities, viz., sweet, sour, salt and bitter. The taste-substances used were: chlornatr., muriatic acid, sacch. alb., sacch., quin. sulph., and quin. pur. In all cases in which chemical combination of the substances was to be strictly avoided quin. sulph. and not quin. pur. was used. The greatest possible chemical purity was sought for these substances, which were dissolved in distilled water. The application was made partly by means of dropping-glass tubes on which a scale graduated by $\frac{1}{10} \mathrm{~cm}$. was engraved, partly by means of soft pointed hair brushes. All disturbing accompanying sensations, not excepting that of temperature, were excluded. The simplest way to accomplish the last was to raise the fluids to be applied to the temperature of the mouth, viz. $37^{\circ} \mathrm{C}$. Between the separate experiments the mouth was rinsed out with pure water of the same temperature, $37^{\circ} \mathrm{C}$. After having trained the subjects, I first examined the cavity of the mouth, with a view to determining what parts were receptive of sensations of taste. These experiments were performed both on children and on adults. Taking into consideration what former investigators have found out the total results of this chapter will be given. 
1. Besides the whole surface of the tongue together with its base and the under surface of its tip-the hard and soft palate, without doubt the arcus glosso-palatinus, the tonsils, the uvula, the isthmus fancium, the inside of the epiglottis and the mucous membrane of the cheeks participate in the sensation of taste.

2. All these parts are sensitive in childhood; in adults the mucous membrane of the cheeks, the middle of the tongue and, with a few exceptions, the hard palate lose their sensitiveness. In some cases the under surface of the tip of the tongue on both sides of the frenulum remains receptive also in adults.

3. The presence of disturbance is accounted for, sometimes by an affection of the cavity of the tympanum, sometimes by individual differences.

It must be remarked that the perceptive faculty of the inner epiglottis was established by Michelson and Langendorff, ${ }^{1}$ that of the mucous membrane of the cheeks in childhood by Urbantschitsch." Concerning the retrogression of certain taste surfaces in adults I must refer the reader to my longer article in which an explanation according to the theory of development is offered and literary references given.

In a further investigation I tested the sensitiveness of the different perceptive parts of the cavity of the mouth, by taking as measure the absolute given for the different qualities of taste, obtaining in this way the following general results:

I. Sensitiveness varies for the different qualities on the different parts of the tongue. Sweet is tasted best on the tip of the tongue, sour on the edge, and bitter at the base, acid equally on the tip and edges, but less at the base.

2. With regard to the values found in an isolated case for the other taste-surfaces, the sensitiveness for sweet and bitter appears in the following order: Soft palate, arcus glosso-palatin., uvula, undersurface of the tip; for sour, arcus glosso-palat., palat. molle, uvula, under-surface of the tip; for salt, palatum molle, under-surface of the tip, arcus glosso-pal., uvula. The values are in part considerably below those noted under $r$. Only on the soft palate does salt reach the normal given.

3. A single investigation showed that in childhood all parts, excepting the tip and edges of the tongue, possessed nearly the same

${ }^{1}$ Centralblatt fur Physiol. 1892. P. 204.

${ }^{2}$ Urbantschitsch, Beobochtungen uber Anomalien des Geschmacks, etc., in Forge von Erkrankungen der Taukenhöhle. I876. - I desire to draw special attention to this interesting work. 
sensitiveness with regard to sweet. The tip and edges were more sensitive.

4. The explanation of the normal condition, as of individual differences is without doubt to be found in the law of adaptation, excepting those cases in which pathological causes, obstructions, etc., appear.

Further, attention was directed to the qualitative conditions of the sensations of taste. These experiments were only made on adults. First, I was enabled to prove that all four above-named qualities are true sensations of taste, also that the sensations of sour and salt must not be excluded from the sphere of taste on account of the accompanying tactile sensations. On the contrary, my investigations led to the conclusion that all our perceptions of taste are accompanied by tactile sensations, although in different degrees. Sweet is accompanied on and near the limen by a sensation of smoothness, at higher intensity by that of slipperiness, at very great intensity by that of scratching and biting. The liminal values of bitter are accompanied by a distinct sensation of greasiness. Even the application of distilled water produced with some of my subjects a distinct perception of taste. Two of them tasted water on the tip of the tongue as sweet, on the edges as sour and sourish, at the base bitter. Others tasted it as bitter in the whole cavity of the mouth, others only bitter at the base and tasteless on the other parts of the tongue. The bitter sensation produced by distilled water accompanied the single sensation called forth by taste-substances often for a time above the limen, so that in this way two sensations arose which I have designated as double-sensations. Even a mechanical stimulus of the base of the tongue with a glassrod produced with me and with many of my subjects a sensation distinctly bitter.

Great influence in the region of taste must be ascribed to association and the effects of contrast. The conditions of contrast I investigated with special care; the total results of which may be given concisely as follows:

I. Contrasting stimuli must be recognized in the sense of taste.

2. Salt contrasts with sweet, salt with sour, sweet with sour.

3. Salt and sweet, and salt and sour contrast both on simultaneous stimulation of corresponding parts of the tongue and on successive stimulation of the same taste-surface. The contrasts of sweet and sour could only be observed in the latter case.

4. Bitter forms an exception, but yet perhaps gives rise to contrasts restricted to individuals.

ThE AUThor.

LEIPZIG. 